\title{
Other articles noted 06 Feb 2004 to 16 Apr 2004
}

list of the journals that are reviewed and the criteria for selecting articles from these journals for inclusion in Evidence-
Based Nursing are included in each issue. The following articles passed all criteria but were not abstracted because, in the
judgment of the editors, their findings were less widely applicable to nursing practice, the topic was of interest to only a
select group of nurse specialists, or the topic was recently addressed in another abstract.

\section{TREATMENT}

Abraham NS, Moayyedi P, Daniels B, et al. The methodological quality of trials affects estimates of treatment efficacy in functional (non-ulcer) dyspepsia. Aliment Pharmacol Ther 2004; 19:631-41.

http://www.ncbi.nlm.nih.gov/entrez/

query.fcgi? $\mathrm{cmd}=$ Retrieve $\& \mathrm{db}=$ pubmed\&dopt $=$ Abstract $\&$ list uids $=15023165$

Adjuik M, Babiker A, Garner P, et al. Artesunate combinations for treatment of malaria: meta-analysis. Lancet 2004;363:9-17.

http://www.ncbi.nlm.nih.gov/entrez/

query.fcgi?cmd $=$ Retrieve $\& \mathrm{db}=$ PubMed\&list_uids $=$ $14723987 \& d o p t=$ Abstract

Aljabri K, Kozak SE, Thompson DM. Addition of pioglitazone or bedtime insulin to maximal doses of sulfonylurea and metformin in type 2 diabetes patients with poor glucose control: a prospective, randomized trial. Am J Med 2004;116:230-5.

http://www.ncbi.nlm.nih.gov/entrez/

query.fcgi? $\mathrm{cmd}=$ Retrieve $\& \mathrm{db}=$ pubmed $\&$ dopt $=$ Abstract $\&$

list_uids $=14969650$

Assendelft W, Morton S, Yu EI, et al. Spinal manipulative therapy for low back pain. Cochrane Database Syst Rev 2004; ( 1):CD000447.

http://www.ncbi.nlm.nih.gov/entrez/

query.fcgi?cmd $=$ Retrieve \&db $=$ PubMed\&list_uids =

14973958\&dopt $=$ Abstract

Avenell A, Handoll HG. Nutritional supplementation for hip fracture aftercare in the elderly. Cochrane Database Syst Rev 2004; (1):CD001880.

http://www.ncbi.nlm.nih.gov/entrez/

query.fcgi? $\mathrm{cmd}=$ Retrieve $\& \mathrm{db}=$ PubMed\&list uids $=$

14973973\&dopt $=$ Abstract

Barden J, Edwards J, Moore RA, et al. Single dose oral rofecoxib for postoperative pain. Cochrane Database Syst Rev 2004; ( 1 ):CD004604.

http://www.ncbi.nlm.nih.gov/entrez/

query.fcgi? $\mathrm{cmd}=$ Retrieve $\& \mathrm{db}=$ pubmed $\&$ dopt $=$ Abstract $\&$ list_uids $=14974074$

Barlow J, Coren E. Parent-training programmes for improving maternal psychosocial health. Cochrane Database Syst Rev 2004; (1):CD002020.

http://www.ncbi.nlm.nih.gov/entrez/

query.fcgi?cmd $=$ Retrieve $\& \mathrm{db}=$ PubMed\&list uids $=$

14973981\&dopt $=$ Abstract

Barrett P, Healy-Farrell L, March JS. Cognitive-behavioral family treatment of childhood obsessive-compulsive disorder: a controlled trial. J Am Acad Child Adolesc Psychiatry 2004:43:46-62. http://www.ncbi.nlm.nih.gov/entrez/

query.fcgi?cmd $=$ Retrieve \&db = PubMed\&list_uids =

14691360\&dopt $=$ Abstract

Bijkerk CJ, Muris JW, Knottnerus JA, et al. Systematic review: the role of different types of fibre in the treatment of irritable bowel syndrome. Aliment Pharmacol Ther 2004: 19:245-51

http://www.ncbi.nlm.nih.gov/entrez/

query.fcgi? $\mathrm{cmd}=$ Retrieve $\& \mathrm{db}=$ pubmed $\&$ dopt $=$ Abstract $\&$ list uids $=14984370$

Bisson JI, Shepherd JP, Joy D, et al. Early cognitivebehavioural therapy for post-traumatic stress symptoms after physical injury. Randomised controlled trial. Br J Psychiatry 2004; 184:63-9.

http://www.ncbi.nlm.nih.gov/entrez/

query.fcgi? $\mathrm{cmd}=$ Retrieve $\& \mathrm{db}=$ PubMed\&list_uids $=$ $14702229 \&$ dopt $=$ Abstract

Brankston GN, Mitchell BF, Ryan EA, et al. Resistance exercise decreases the need for insulin in overweight women with gestational diabetes mellitus. Am J Obstet Gynecol 2004; 190:188-93.

http://www.ncbi.nlm.nih.gov/entrez/

query.fcgi? $\mathrm{cmd}=$ Retrieve $\& \mathrm{db}=$ PubMed\&list_uids $=$

$14749658 \&$ dopt $=$ Abstract

Bruce ML, Ten Have TR, Reynolds CF 3rd, et al. Reducing suicidal ideation and depressive symptoms in depressed older primary care patients: a randomized controlled trial. JAMA 2004;291:1081-91.

http://www.ncbi.nlm.nih.gov/entrez/

query.fcgi? $\mathrm{cmd}=$ Retrieve $\& \mathrm{db}=$ pubmed $\&$ dopt $=$ Abstract $\&$ list_uids $=14996777$

Bueving HJ, Bernsen RM, de Jongste JC, et al. Influenza vaccination in children with asthma: randomized doubleblind placebo-controlled trial. Am J Respir Crit Care Med 2004; 169:488-93.

http://www.ncbi.nlm.nih.gov/entrez/ query.fcgi? $\mathrm{cmd}=$ Retrieve $\& \mathrm{db}=$ pubmed $\&$ dopt $=$ Abstract $\&$ list uids $=14656755$

Burton M, Dorée C. Interventions for recurrent idiopathic epistaxis (nosebleeds) in children. Cochrane Database Syst Rev 2004; ( 1):CD004461.

http://www.ncbi.nlm.nih.gov/entrez/

query.fcgi? $\mathrm{cmd}=$ Retrieve $\& \mathrm{db}=$ PubMed\&list_uids $=$ 14974069\&dopt $=$ Abstract

Challis D, Clarkson P, Williamson $\mathrm{J}$, et al. The value of specialist clinical assessment of older people prior to entry to care homes. Age Ageing 2004;33:25-34.

http://www.ncbi.nlm.nih.gov/entrez/

query.fcgi? $\mathrm{cmd}=$ Retrieve \&db $=$ PubMed\&list_uids $=$ 14695860\&dopt $=$ Abstract 
2 of 13

Cheng KK, Chang AM. Palliation of oral mucositis symptoms in pediatric patients treated with cancer chemotherapy. Cancer Nurs 2003;26:476-84.

http://www.ncbi.nlm.nih.gov/entrez/

query.fcgi?cmd $=$ Retrieve $\& d b=$ pubmed\&dopt $=$ Abstract $\&$ list uids $=15022979$

Chlebowski RT, Wactawski-Wende J, Ritenbaugh C, et al Estrogen plus progestin and colorectal cancer in postmenopausal women. $N$ Engl J Med 2004;350:991-1004.

http://www.ncbi.nlm.nih.gov/entrez/

query.fcgi? $\mathrm{cmd}=$ Retrieve $\& \mathrm{db}=$ pubmed $\&$ dopt $=$ Abstract $\&$ list_uids = 14999111

Christ-Crain M, Jaccard-Stolz D, Bingisser R, et al. Effect of procalcitonin-guided treatment on antibiotic use and outcome in lower respiratory tract infections: cluster-randomised, single-blinded intervention trial. Lancet 2004;363:600-

http://www.ncbi.nlm.nih.gov/entrez/

query.fcgi?cmd $=$ Retrieve $\& \mathrm{db}=$ pubmed $\&$ dopt $=$ Abstract $\&$ list_uids $=14987884$

Clark HF, Bernstein DI, Dennehy PH, et al. Safety, efficacy, and immunogenicity of a live, quadrivalent human-bovine reassortant rotavirus vaccine in healthy infants. $J$ Pediatr 2004; 144:184-90

http://www.ncbi.nlm.nih.gov/entrez/

query.fcgi $\mathrm{cmd}=$ Retrieve $\& \mathrm{db}=$ pubmed $\& \mathrm{dopt}=$ Abstract $\&$

list_uids $=14760258$

Clarkson J, Worthington $\mathrm{H}$, Eden O. Interventions for treating oral candidiasis for patients with cancer receiving treatment. Cochrane Database Syst Rev 2004;(1):CD001972.

http://www.ncbi.nlm.nih.gov/entrez/

query.fcgi?cmd $=$ Retrieve \&db $=$ PubMed\&list_uids $=$

14973977\&dopt $=$ Abstract

Conner, JM, Soll R, Edwards W. Topical ointment for preventing infection in preterm infants. Cochrane Database Syst Rev 2004; (1):CD001150.

http://www.ncbi.nlm.nih.gov/entrez/

query.fcgi? $\mathrm{cmd}=$ Retrieve $\& \mathrm{db}=$ PubMed\&list_uids $=$

14973963\&dopt $=$ Abstract

Corey L, Wald A, Patel R, et al. Once-daily valacyclovir to reduce the risk of transmission of genital herpes. $N$ Engl J Med 2004;350:11-20.

http://www.ncbi.nlm.nih.gov/entrez/

query.fcgi?cmd $=$ Retrieve \&db $=$ PubMed\&list_uids $=$

$14702423 \&$ dopt $=$ Abstract

Correll CU, Leucht S, Kane JM. Lower risk for tardive dyskinesia associated with second-generation antipsychotics: a systematic review of l-year studies. Am J Psychiatry 2004;161:414-25.

http://www.ncbi.nlm.nih.gov/entrez/

query.fcgi?cmd $=$ Retrieve $\& \mathrm{db}=$ pubmed $\& \mathrm{dopt}=$ Abstract $\&$ list_uids $=14992963$

Cummings JL, Schneider L, Tariot PN, et al. Reduction of behavioral disturbances and caregiver distress by galantamine in patients with Alzheimer's disease. Am J Psychiatry 2004; 161:532-8.

http://www.ncbi.nlm.nih.gov/entrez/

query.fcgi? $\mathrm{cmd}=$ Retrieve $\& \mathrm{db}=$ pubmed $\& \mathrm{dopt}=$ Abstract $\&$ list_uids $=14992980$

Dailey GE 3rd, Noor MA, Park JS, et al. Glycemic control with glyburide/metformin tablets in combination with rosiglita- zone in patients with type 2 diabetes: a randomized, doubleblind trial. Am J Med 2004;116:223-9.

http://www.ncbi.nlm.nih.gov/entrez/

query.fcgi?cmd $=$ Retrieve $\& \mathrm{db}=$ pubmed $\&$ dopt $=$ Abstract $\&$ list uids $=14969649$

Day E, Bentham P, Callaghan R, et al. Thiamine for WernickeKorsakoff Syndrome in people at risk from alcohol abuse. Cochrane Database Syst Rev 2004;(1):CD004033.

http://www.ncbi.nlm.nih.gov/entrez/

query.fcgi? $\mathrm{cmd}=$ Retrieve $\& \mathrm{db}=$ PubMed\&list_uids $=$

14974055\&dopt $=$ Abstract

Dean T, Dewey A, Bara A, et al. Azathioprine as an oral corticosteroid sparing agent for asthma. Cochrane Database Syst Rev 2004; ( 1):CD003270

http://www.ncbi.nlm.nih.gov/entrez/

query.fcgi? $\mathrm{cmd}=$ Retrieve $\& \mathrm{db}=$ PubMed\&list_uids $=$

$14974011 \&$ dopt $=$ Abstract

Dey P, Simpson CW, Collins SI, et al. Implementation of RCGP guidelines for acute low back pain: a cluster randomised controlled trial. Br J Gen Pract 2004;54:33-7. http://www.ncbi.nlm.nih.gov/entrez/

query.fcgi? $\mathrm{cmd}=$ Retrieve $\& \mathrm{db}=$ pubmed $\&$ dopt $=$ Abstract $\&$ list uids $=14965404$

Dombrowski MP, Schatz M, Wise R, et al. Randomized trial of inhaled beclomethasone dipropionate versus theophylline for moderate asthma during pregnancy. Am J Obstet Gynecol 2004; 190:737-44.

http://www.ncbi.nlm.nih.gov/entrez/

query.fcgi? $\mathrm{cmd}=$ Retrieve $\& \mathrm{db}=$ pubmed \&dopt $=$ Abstract $\&$ list uids $=15042007$

Donnelly M, Power M, Russell M, et al. Randomized controlled trial of an early discharge rehabilitation service: the Belfast Community Stroke Trial. Stroke 2004;35:127-33. http://www.ncbi.nlm.nih.gov/entrez/ query.fcgi?cmd $=$ Retrieve $\& \mathrm{db}=$ PubMed\&list_uids $=$ $14671238 \&$ dopt $=$ Abstract

Dukas L, Bischoff HA, Lindpaintner LS, et al. Alfacalcidol reduces the number of fallers in a community-dwelling elderly population with a minimum calcium intake of more than $500 \mathrm{mg}$ daily. J Am Geriatr Soc 2004;52:230-6.

http://www.ncbi.nlm.nih.gov/entrez/

query.fcgi? $\mathrm{cmd}=$ Retrieve $\& \mathrm{db}=$ pubmed $\& \mathrm{dopt}=$ Abstract $\&$ list_uids $=14728632$

Ekeland E, Heian F, Hagen K, et al. Exercise to improve selfesteem in children and young people. Cochrane Database Syst Rev 2004; (1):CD003683.

http://www.ncbi.nlm.nih.gov/entrez/

query.fcgi? $\mathrm{cmd}=$ Retrieve $\& \mathrm{db}=$ PubMed\&list_uids $=$

14974029\&dopt $=$ Abstract

Elkins M, Jones A, van der Schans C. Positive expiratory pressure physiotherapy for airway clearance in people with cystic fibrosis. Cochrane Database Syst Rev 2004;(1):CD003147. http://www.ncbi.nlm.nih.gov/entrez/

query.fcgi?cmd $=$ Retrieve \&db $=$ PubMed\&list_uids $=$

$14974007 \&$ dopt $=$ Abstract

Emkey R, Rosenthal N, Wu SC, et al. Efficacy and safety of tramadol/acetaminophen tablets (Ultracet) as add-on therapy for osteoarthritis pain in subjects receiving a COX-2 nonsteroidal antiinflammatory drug: a multicenter, randomized, double-blind, placebo-controlled trial. J Rheumatol 2004;31:150-6. 
http://www.ncbi.nlm.nih.gov/entrez/ query.fcgi?cmd $=$ Retrieve $\&$ db $=$ PubMed\&list_uids $=$ 14705234\&dopt $=$ Abstract

Emmen MJ, Schippers GM, Bleijenberg G, et al. Effectiveness of opportunistic brief interventions for problem drinking in a general hospital setting: systematic review. BMJ 2004;328:318-22.

http://www.ncbi.nlm.nih.gov/entrez/

query.fcgi? $\mathrm{cmd}=$ Retrieve $\& \mathrm{db}=$ PubMed\&list_uids $=$ $14729657 \& d o p t=$ Abstract

Ferreira ML, Ferreira PH, Latimer J, et al. Efficacy of spinal manipulative therapy for low back pain of less than three months' duration. J Manipulative Physiol Ther 2003;26:593601.

http://www.ncbi.nlm.nih.gov/entrez/

query.fcgi?cmd $=$ Retrieve \&db $=$ PubMed\&list_uids $=$

$14673408 \& d o p t=$ Abstract

Gallefoss F. The effects of patient education in COPD in a l-year follow-up randomised, controlled trial. Patient Educ Couns 2004;52:259-66.

http://www.ncbi.nlm.nih.gov/entrez/

query.fcgi? $\mathrm{cmd}=$ Retrieve $\& \mathrm{db}=$ pubmed $\&$ dopt $=$ Abstract $\&$

list_uids $=14998595$

Geddes JR, Burgess S, Hawton K, et al. Long-term lithium therapy for bipolar disorder: systematic review and metaanalysis of randomized controlled trials. Am J Psychiatry 2004; 161:217-22.

http://www.ncbi.nlm.nih.gov/entrez/

query.fcgi? $\mathrm{cmd}=$ Retrieve $\& \mathrm{db}=$ pubmed $\&$ dopt $=$ Abstract $\&$

list_uids $=14754766$

Gillies D, O'Riordan E, Carr D, et al. Central venous catheter dressings: a systematic review. J Adv Nurs 2003;44:623-32. http://www.ncbi.nlm.nih.gov/entrez/ query.fcgi?cmd $=$ Retrieve \&db $=$ PubMed\&list_uids $=$ $14651685 \&$ dopt $=$ Abstract

Glazener C, Evans J, Peto R. Complex behavioural and educational interventions for nocturnal enuresis in children. Cochrane Database Syst Rev 2004;(1):CD004668.

http://www.ncbi.nlm.nih.gov/entrez/

query.fcgi?cmd $=$ Retrieve \&db $=$ PubMed\&list uids $=$

$14974076 \& d o p t=$ Abstract

Gosselin R, Roberts I, Gillespie W. Antibiotics for preventing infection in open limb fractures. Cochrane Database Syst Rev 2004; (1):CD003764.

http://www.ncbi.nlm.nih.gov/entrez/

query.fcgi? $\mathrm{cmd}=$ Retrieve $\& \mathrm{db}=$ PubMed\&list uids $=$

14974035\&dopt $=$ Abstract

Gowers S, Bryant-Waugh R. Management of child and adolescent eating disorders: the current evidence base and future directions. J Child Psychol Psychiatry 2004;45: 63-83.

http://www.ncbi.nlm.nih.gov/entrez/

query.fcgi?cmd $=$ Retrieve\&db $=$ PubMed\&list_uids $=$

$14959803 \&$ dopt $=$ Abstract

Gruen RL, Weeramanthri TS, Knight SE, et al. Specialist outreach clinics in primary care and rural hospital settings. Cochrane Database Syst Rev 2004; (1):CD003798.

http://www.ncbi.nlm.nih.gov/entrez/

query.fcgi? $\mathrm{cmd}=$ Retrieve $\& \mathrm{db}=$ pubmed $\&$ dopt $=$ Abstract $\&$

list_uids $=14974038$
Haines TP, Bennell KL, Osborne RH, et al. Effectiveness of targeted falls prevention programme in subacute hospital setting: randomised controlled trial. BMJ 2004;328:676-81. http://www.ncbi.nlm.nih.gov/entrez/ query.fcgi?cmd $=$ Retrieve $\& \mathrm{db}=$ pubmed $\&$ dopt $=$ Abstract $\&$ list_uids $=15031238$

Halpern MT, Schmier JK, Van Kerkhove MD, et al. Impact of long-term inhaled corticosteroid therapy on bone mineral density: results of a meta-analysis. Ann Allergy Asthma Immunol 2004;92:201-7.

http://www.ncbi.nlm.nih.gov/entrez/

query.fcgi?cmd $=$ Retrieve $\& \mathrm{db}=$ pubmed $\&$ dopt $=$ Abstract $\&$ list_uids $=14989387$

Hambidge SJ, Davidson AJ, Phibbs SL, et al. Strategies to improve immunization rates and well-child care in a disadvantaged population: a cluster randomized controlled trial. Arch Pediatr Adolesc Med 2004;158:162-9.

http://www.ncbi.nlm.nih.gov/entrez/

query.fcgi? $\mathrm{cmd}=$ Retrieve $\& \mathrm{db}=$ pubmed $\&$ dopt $=$ Abstract $\&$ list uids $=14757608$

Hammond A, Young A, Kidao R. A randomised controlled trial of occupational therapy for people with early rheumatoid arthritis. Ann Rheum Dis 2004;63:23-30.

http://www.ncbi.nlm.nih.gov/entrez/

query.fcgi $\mathrm{cmd}=$ Retrieve $\& \mathrm{db}=$ pubmed $\&$ dopt $=$ Abstract $\&$ list_uids $=14672887$

Hanifin JM, Cooper KD, Ho VC, et al. Guidelines of care for atopic dermatitis. J Am Acad Dermatol 2004;50:391-404

http://www.ncbi.nlm.nih.gov/entrez/

query.fcgi? $\mathrm{cmd}=$ Retrieve $\& \mathrm{db}=$ pubmed $\&$ dopt $=$ Abstract $\&$ list_uids $=14988682$

Hansen JJ, Ulmer BJ, Rex DK. Technical performance of colonoscopy in patients sedated with nurse-administered propofol. Am J Gastroenterol 2004;99:52-6.

http://www.ncbi.nlm.nih.gov/entrez/

query.fcgi? $\mathrm{cmd}=$ Retrieve $\& \mathrm{db}=$ pubmed $\&$ dopt $=$ Abstract $\&$ list uids $=14687141$

Harrington J, Noble LM, Newman SP. Improving patients communication with doctors: a systematic review of intervention studies. Patient Educ Couns 2004;52:7-16.

http://www.ncbi.nlm.nih.gov/entrez/

query.fcgi? $\mathrm{cmd}=$ Retrieve\&db $=$ PubMed\&list uids $=$ $14729285 \&$ dopt $=$ Abstract

Harrison TW, Oborne J, Newton S, et al. Doubling the dose of inhaled corticosteroid to prevent asthma exacerbations: randomised controlled trial. Lancet 2004;363:271-5. http://www.ncbi.nlm.nih.gov/entrez/ query.fcgi? $\mathrm{cmd}=$ Retrieve $\& \mathrm{db}=$ PubMed\&list_uids $=$ 14751699\&dopt $=$ Abstract

Hartling L, Wiebe N, Russell $\mathrm{K}$, et al. Epinephrine for bronchiolitis. Cochrane Database Syst Rev 2004;(1):CD003123. http://www.ncbi.nlm.nih.gov/entrez/

query.fcgi?cmd $=$ Retrieve $\& \mathrm{db}=$ PubMed\&list_uids $=$ $14974006 \&$ dopt $=$ Abstract

Hatsukami DK, Rennard S, Patel MK, et al. Effects of sustained-release bupropion among persons interested in reducing but not quitting smoking. Am J Med 2004;116: $151-7$.

http://www.ncbi.nlm.nih.gov/entrez/

query.fcgi? $\mathrm{cmd}=$ Retrieve $\& \mathrm{db}=$ pubmed $\&$ dopt $=$ Abstract $\&$ list_uids $=14749158$ 
4 of 13

Hayden FG, Belshe R, Villanueva C, et al. Management of influenza in households: a prospective, randomized comparison of oseltamivir treatment with or without postexposure prophylaxis. $J$ Infect Dis 2004;189:440-9.

http://www.ncbi.nlm.nih.gov/entrez/

query.fcgi? $\mathrm{cmd}=$ Retrieve $\& \mathrm{db}=$ pubmed $\&$ dopt $=$ Abstract $\&$ list_uids $=14745701$

Hayden JT, Smith N, Woolf DA, et al. A randomised crossover trial of facemask efficacy. Arch Dis Child 2004;89:72-3. http://www.ncbi.nlm.nih.gov/entrez/

query.fcgi?cmd $=$ Retrieve \&db $=$ PubMed\&list_uids $=$ 14709514\&dopt $=$ Abstract

Heinen MM, van Achterberg T, op Reimer WS, et al. Venous leg ulcer patients: a review of the literature on lifestyle and pain-related interventions. J Clin Nurs 2004;13:355-66. http://www.ncbi.nlm.nih.gov/entrez/ query.fcgi? $\mathrm{cmd}=$ Retrieve $\& \mathrm{db}=$ pubmed $\&$ dopt $=$ Abstract $\&$ list_uids $=15009338$

Holloway E, Ram F. Breathing exercises for asthma. Cochrane Database Syst Rev 2004;(1):CD001277.

http://www.ncbi.nlm.nih.gov/entrez/

query.fcgi?cmd $=$ Retrieve $\& \mathrm{db}=$ PubMed\&list_uids $=$ 14973966\&dopt $=$ Abstract

Hooper L, Bartlett C, Davey Smith G, et al. Advice to reduce dietary salt for prevention of cardiovascular disease. Cochrane Database Syst Rev 2004; ( 1):CD003656.

http://www.ncbi.nlm.nih.gov/entrez/

query.fcgi? cmd $=$ Retrieve \&db $=$ PubMed\&list_uids $=$ 14974027\&dopt $=$ Abstract

Hsia J, Criqui MH, Rodabough RJ, et al. Estrogen plus progestin and the risk of peripheral arterial disease: the Women's Health Initiative. Circulation 2004;109:620-6. http://www.ncbi.nlm.nih.gov/entrez/

query.fcgi? $\mathrm{cmd}=$ Retrieve $\& \mathrm{db}=$ pubmed $\& \mathrm{dopt}=$ Abstract $\&$ list_uids $=14769684$

Huey SJ Jr, Henggeler SW, Rowland MD, et al. Multisystemic therapy effects on attempted suicide by youths presenting psychiatric emergencies. J Am Acad Child Adolesc Psychiatry 2004;43:183-90.

http://www.ncbi.nlm.nih.gov/entrez/

query.fcgi cmd $=$ Retrieve $\& \mathrm{db}=$ pubmed $\&$ dopt $=$ Abstract $\&$ list_uids $=14726725$

Hurwitz EL, Morgenstern H, Vassilaki M, et al. Adverse reactions to chiropractic treatment and their effects on satisfaction and clinical outcomes among patients enrolled in the UCLA Neck Pain Study. J Manipulative Physiol Ther 2004;27:16-25.

http://www.ncbi.nlm.nih.gov/entrez/

query.fcgi? $\mathrm{cmd}=$ Retrieve $\& \mathrm{db}=$ pubmed\&dopt $=$ Abstract $\&$ list uids $=14739870$

Jarjoura D, Polen A, Baum E, et al. Effectiveness of screening and treatment for depression in ambulatory indigent patients. J Gen Intern Med 2004;19:78-84.

http://www.ncbi.nlm.nih.gov/entrez/

query.fcgi? $\mathrm{cmd}=$ Retrieve $\& \mathrm{db}=$ pubmed $\&$ dopt $=$ Abstract $\&$ list_uids $=14748864$

Jepson R, Mihaljevic L, Craig J. Cranberries for preventing urinary tract infections. Cochrane Database Syst Rev 2004; (1):CD001321.

http://www.ncbi.nlm.nih.gov/entrez/

query.fcgi? $\mathrm{cmd}=$ Retrieve $\& \mathrm{db}=$ PubMed\&list uids $=$

14973968\&dopt $=$ Abstract
Jürgens G, Graudal N. Effects of low sodium diet versus high sodium diet on blood pressure, renin, aldosterone, catecholamines, cholesterols, and triglyceride. Cochrane Database Syst Rev 2004; (1):CD004022.

http://www.ncbi.nlm.nih.gov/entrez/

query.fcgi?cmd $=$ Retrieve $\&$ db $=$ PubMed\&list_uids $=$

14974053\&dopt $=$ Abstract

Keystone EC, Schiff MH, Kremer JM, et al. Once-weekly administration of $50 \mathrm{mg}$ etanercept in patients with active rheumatoid arthritis: results of a multicenter, randomized, double-blind, placebo-controlled trial. Arthritis Rheum 2004;50:353-63.

http://www.ncbi.nlm.nih.gov/entrez/

query.fcgi?cmd $=$ Retrieve $\& \mathrm{db}=$ pubmed $\&$ dopt $=$ Abstract $\&$ list uids $=14872476$

King VJ, Viswanathan M, Bordley WC, et al. Pharmacologic treatment of bronchiolitis in infants and children: a systematic review. Arch Pediatr Adolesc Med 2004;158: 127-37.

http://www.ncbi.nlm.nih.gov/entrez/

query.fcgi? $\mathrm{cmd}=$ Retrieve $\& \mathrm{db}=$ pubmed $\&$ dopt $=$ Abstract $\&$ list uids $=14757604$

Klareskog L, van der Heijde D, de Jager JP, et al. Therapeutic effect of the combination of etanercept and methotrexate compared with each treatment alone in patients with rheumatoid arthritis: double-blind randomised controlled trial. Lancet 2004;363:675-81.

http://www.ncbi.nlm.nih.gov/entrez/

query.fcgi? $\mathrm{cmd}=$ Retrieve $\& \mathrm{db}=$ pubmed $\&$ dopt $=$ Abstract $\&$ list_uids $=15001324$

Koivunen P, Uhari M, Luotonen J, et al. Adenoidectomy versus chemoprophylaxis and placebo for recurrent acute otitis media in children aged under 2 years: randomised controlled trial. BMJ 2004;328:487-91.

http://www.ncbi.nlm.nih.gov/entrez/

query.fcgi? $\mathrm{cmd}=$ Retrieve $\& \mathrm{db}=$ pubmed $\&$ dopt $=$ Abstract $\&$ list uids $=14769785$

Kulier R, Gülmezoglu A, Hofmeyr G, et al. Medical methods for first trimester abortion. Cochrane Database Syst Rev 2004; ( 1):CD002855.

http://www.ncbi.nlm.nih.gov/entrez/

query.fcgi?cmd $=$ Retrieve $\& \mathrm{db}=$ PubMed\&list_uids $=$

$14973995 \&$ dopt $=$ Abstract

Kuschel C, Harding J. Multicomponent fortified human milk for promoting growth in preterm infants. Cochrane Database Syst Rev 2004; ( 1):CD000343.

http://www.ncbi.nlm.nih.gov/entrez/

query.fcgi?cmd $=$ Retrieve $\& \mathrm{db}=$ PubMed\&list_uids $=$

14973953\&dopt $=$ Abstract

Larson EL, Lin SX, Gomez-Pichardo C, et al. Effect of antibacterial home cleaning and handwashing products on infectious disease symptoms: a randomized, double-blind trial. Ann Intern Med 2004; 140:321-9.

http://www.ncbi.nlm.nih.gov/entrez/

query.fcgi?cmd $=$ Retrieve $\& \mathrm{db}=$ pubmed $\&$ dopt $=$ Abstract $\&$ list uids $=14996673$

Lawes CM, Bennett DA, Feigin VL, et al. Blood pressure and stroke: an overview of published reviews. Stroke 2004;35:77685.

http://www.ncbi.nlm.nih.gov/entrez/

query.fcgi? $\mathrm{cmd}=$ Retrieve $\& \mathrm{db}=$ pubmed $\&$ dopt $=$ Abstract $\&$ list_uids $=14976329$ 
Lerman C, Kaufmann V, Rukstalis M, et al. Individualizing nicotine replacement therapy for the treatment of tobacco dependence: a randomized trial. Ann Intern Med 2004; 140:426-33.

http://www.ncbi.nlm.nih.gov/entrez/

query.fcgi? $\mathrm{cmd}=$ Retrieve $\& \mathrm{db}=$ pubmed $\&$ dopt $=$ Abstract $\&$ list_uids $=15023708$

Liberati A, D'Amico R, Pifferi, et al. Antibiotic prophylaxis to reduce respiratory tract infections and mortality in adults receiving intensive care. Cochrane Database Syst Rev 2004; (1):CD000022.

http://www.ncbi.nlm.nih.gov/entrez/

query.fcgi? $\mathrm{cmd}=$ Retrieve $\& \mathrm{db}=$ PubMed\&list_uids $=$ 14973945\&dopt $=$ Abstract

Liddle SD, Baxter GD, Gracey JH. Exercise and chronic low back pain: what works? Pain 2004;107:176-90.

http://www.ncbi.nlm.nih.gov/entrez/

query.fcgi? $\mathrm{cmd}=$ Retrieve $\& \mathrm{db}=$ pubmed \&dopt $=$ Abstract $\&$ list_uids $=14715404$

Loeb MB, Molloy D, Smieja M, et al. A randomized, controlled trial of doxycycline and rifampin for patients with Alzheimer's disease. J Am Geriatr Soc 2004;52:381-7.

http://www.ncbi.nlm.nih.gov/entrez/

query.fcgi? $\mathrm{cmd}=$ Retrieve $\& \mathrm{db}=$ pubmed $\&$ dopt $=$ Abstract $\&$

list_uids $=14962152$

Ma SH, Teasdale JD. Mindfulness-based cognitive therapy for depression: replication and exploration of differential relapse prevention effects. J Consult Clin Psychol 2004;72:31-40.

http://www.ncbi.nlm.nih.gov/entrez/

query.fcgi? $\mathrm{cmd}=$ Retrieve $\& \mathrm{db}=$ pubmed \&dopt $=$ Abstract $\&$ list_uids $=14756612$

Madisch A, Holtmann G, Plein K, et al. Treatment of irritable bowel syndrome with herbal preparations: results of a double-blind, randomized, placebo-controlled, multi-centre trial. Aliment Pharmacol Ther 2004;19:271-9.

http://www.ncbi.nlm.nih.gov/entrez/

query.fcgi? $\mathrm{cmd}=$ Retrieve $\& \mathrm{db}=$ pubmed \&dopt $=$ Abstract $\&$ list uids $=14984373$

Marras TK, Nopmaneejumruslers C, Chan CK. Efficacy of exclusively oral antibiotic therapy in patients hospitalized with nonsevere community-acquired pneumonia: a retrospective study and meta-analysis. Am J Med 2004;116:385-93. http://www.ncbi.nlm.nih.gov/entrez/

query.fcgi? $\mathrm{cmd}=$ Retrieve $\& \mathrm{db}=$ pubmed\&dopt $=$ Abstract $\&$ list_uids $=15006587$

McCarney R, Brinkhaus B, Lasserson T, et al. Acupuncture for chronic asthma. Cochrane Database Syst Rev 2004;(1): CD000008

http://www.ncbi.nlm.nih.gov/entrez/

query.fcgi?cmd = Retrieve\&db = PubMed\&list uids = 14973944\&dopt $=$ Abstract

McCarney R, Linde K, Lasserson T. Homeopathy for chronic asthma. Cochrane Database Syst Rev 2004;(1):CD000353. http://www.ncbi.nlm.nih.gov/entrez/

query.fcgi?cmd $=$ Retrieve $\& \mathrm{db}=$ PubMed\&list_uids $=$ $14973954 \&$ dopt $=$ Abstract

McClellan JM, Werry JS. Evidence-based treatments in child and adolescent psychiatry: an inventory. J Am Acad Child Adolesc Psychiatry 2003;42:1388-400.

http://www.ncbi.nlm.nih.gov/entrez/

query.fcgi?cmd $=$ Retrieve $\& \mathrm{db}=$ PubMed\&list_uids $=$

14627873\&dopt $=$ Abstract
McDonald S, Hetrick S, Green S. Pre-operative education for hip or knee replacement. Cochrane Database Syst Rev 2004; (1):CD003526.

http://www.ncbi.nlm.nih.gov/entrez/

query.fcgi?cmd $=$ Retrieve $\& \mathrm{db}=$ pubmed $\&$ dopt $=$ Abstract $\&$

list_uids $=14974019$

Meng CF, Wang D, Ngeow J, et al. Acupuncture for chronic low back pain in older patients: a randomized, controlled trial. Rheumatology (Oxford) 2003;42:1508-17.

http://www.ncbi.nlm.nih.gov/entrez/

query.fcgi? $\mathrm{cmd}=$ Retrieve $\& \mathrm{db}=$ PubMed\&list_uids $=$ $12890859 \&$ dopt $=$ Abstract

Miranda-Filho Dde B, Ximenes RA, Barone AA, et al. Randomised controlled trial of tetanus treatment with antitetanus immunoglobulin by the intrathecal or intramuscular route. BMJ 2004;328:615-8.

http://www.ncbi.nlm.nih.gov/entrez/

query.fcgi? $\mathrm{cmd}=$ Retrieve $\& \mathrm{db}=$ pubmed $\&$ dopt $=$ Abstract $\&$ list_uids $=15003976$

Montgomery P, Stores G, Wiggs L. The relative efficacy of two brief treatments for sleep problems in young learning disabled (mentally retarded) children: a randomised controlled trial. Arch Dis Child 2004;89:125-30.

http://www.ncbi.nlm.nih.gov/entrez/

query.fcgi? $\mathrm{cmd}=$ Retrieve $\& \mathrm{db}=$ pubmed $\&$ dopt $=$ Abstract $\&$ list_uids $=14736626$

Morin CM, Bastien C, Guay B, et al. Randomized clinical trial of supervised tapering and cognitive behavior therapy to facilitate benzodiazepine discontinuation in older adults with chronic insomnia. Am J Psychiatry 2004; 161:332-42.

http://www.ncbi.nlm.nih.gov/entrez/

query.fcgi? $\mathrm{cmd}=$ Retrieve $\& \mathrm{db}=$ pubmed $\&$ dopt $=$ Abstract $\&$ list_uids $=14754783$

Mull CC, Scarfone RJ, Ferri LR, et al. A randomized trial of nebulized epinephrine vs albuterol in the emergency department treatment of bronchiolitis. Arch Pediatr Adolesc Med 2004; 158:113-8.

http://www.ncbi.nlm.nih.gov/entrez/

query.fcgi? $\mathrm{cmd}=$ Retrieve $\& \mathrm{db}=$ pubmed $\&$ dopt $=$ Abstract $\&$ list_uids $=14757602$

Nelson HD, Nygren P, McInerney Y, et al. Screening women and elderly adults for family and intimate partner violence: a review of the evidence for the US Preventive Services Task Force. Ann Intern Med 2004; 140:387-96.

http://www.ncbi.nlm.nih.gov/entrez/

query.fcgi?cmd $=$ Retrieve $\& \mathrm{db}=$ pubmed $\&$ dopt $=$ Abstract $\&$ list_uids $=14996681$

$\mathrm{Ng} \mathrm{KH}$, Chong D, Wong CK, et al. Central nervous system side effects of first- and second-generation antihistamines in school children with perennial allergic rhinitis: a randomized, double-blind, placebo-controlled comparative study. Pediatrics 2004;113:el16-21.

http://www.ncbi.nlm.nih.gov/entrez/

query.fcgi? $\mathrm{cmd}=$ Retrieve $\& \mathrm{db}=$ pubmed $\&$ dopt $=$ Abstract $\&$ list_uids $=14754980$

Norman SA, Lumley MA, Dooley JA, et al. For whom does it work? Moderators of the effects of written emotional disclosure in a randomized trial among women with chronic pelvic pain. Psychosom Med 2004;66:174-83.

http://www.ncbi.nlm.nih.gov/entrez/

query.fcgi? $\mathrm{cmd}=$ Retrieve $\& \mathrm{db}=$ pubmed $\&$ dopt $=$ Abstract $\&$ list_uids $=15039501$ 
6 of 13

Ohlsson A, Lacy J. Intravenous immunoglobulin for preventing infection in preterm and/or low-birth-weight infants. Cochrane Database Syst Rev 2004;(1):CD000361.

http://www.ncbi.nlm.nih.gov/entrez/

query.fcgi?cmd $=$ Retrieve \&db = PubMed\&list_uids $=$ 14973955\&dopt $=$ Abstract

Peacock SC, Forbes DA. Interventions for caregivers of persons with dementia: a systematic review. Can J Nurs Res 2003;35:88-107.

http://www.ncbi.nlm.nih.gov/entrez/

query.fcgi?cmd $=$ Retrieve\&db $=$ pubmed\&dopt $=$ Abstract $\&$ list_uids $=14746123$

Petrie KJ, Fontanilla I, Thomas MG, et al. Effect of written emotional expression on immune function in patients with human immunodeficiency virus infection: a randomized trial Psychosom Med 2004;66:272-5.

http://www.ncbi.nlm.nih.gov/entrez/

query.fcgi?cmd $=$ Retrieve $\& \mathrm{db}=$ pubmed \&dopt $=$ Abstract $\&$ list_uids $=15039514$

Piepoli MF, Davos C, Francis DP, et al; ExTraMATCH Collaborative. Exercise training meta-analysis of trials in patients with chronic heart failure (ExTraMATCH). BMJ 2004;328:189.

http://www.ncbi.nlm.nih.gov/entrez/

query.fcgi?cmd $=$ Retrieve $\& \mathrm{db}=$ PubMed\&list_uids $=$

14729656\&dopt $=$ Abstract

Pittler M, Ernst E. Feverfew for preventing migraine. Cochrane Database Syst Rev 2004; ( 1):CD002286.

http://www.ncbi.nlm.nih.gov/entrez/

query.fcgi?cmd $=$ Retrieve\&db $=$ PubMed\&list_uids $=$

14973986\&dopt $=$ Abstract

Powe BD, Finnie R. Cancer fatalism: the state of the science. Cancer Nurs 2003;26:454-65.

Price JD, Sear JW, Venn RM. Perioperative fluid volume optimization following proximal femoral fracture. Cochrane Database Syst Rev 2004;(1):CD003004

http://www.ncbi.nlm.nih.gov/entrez/

query.fcgi? $\mathrm{cmd}=$ Retrieve $\& \mathrm{db}=$ pubmed $\&$ dopt $=$ Abstract $\&$

list_uids $=14974001$

Puolakka K, Kautiainen H, Möttönen T, et al. Impact of initial aggressive drug treatment with a combination of diseasemodifying antirheumatic drugs on the development of work disability in early rheumatoid arthritis: a five-year randomized followup trial. Arthritis Rheum 2004;50:55-62.

http://www.ncbi.nlm.nih.gov/entrez/

query.fcgi?cmd $=$ Retrieve $\& \mathrm{db}=$ pubmed $\&$ dopt $=$ Abstract $\&$ list uids $=14730599$

Quinlan DJ, McQuillan A, Eikelboom JW. Low-molecularweight heparin compared with intravenous unfractionated heparin for treatment of pulmonary embolism: a metaanalysis of randomized, controlled trials. Ann Intern Med 2004; 140:175-83.

http://www.ncbi.nlm.nih.gov/entrez/

query.fcgi?cmd $=$ Retrieve $\& \mathrm{db}=$ pubmed $\&$ dopt $=$ Abstract $\&$ list_uids $=14757615$

Ram F, Picot J, Lightowler J, et al. Non-invasive positive pressure ventilation for treatment of respiratory failure due to exacerbations of chronic obstructive pulmonary disease. Cochrane Database Syst Rev 2004;(1):CD004104.

http://www.ncbi.nlm.nih.gov/entrez/

query.fcgi? $\mathrm{cmd}=$ Retrieve $\& \mathrm{db}=$ PubMed\&list uids $=$

14974057\&dopt $=$ Abstract
Ramchandani P, Jones DP. Treating psychological symptoms in sexually abused children: from research findings to service provision. Br J Psychiatry 2003;183:484-90.

http://www.ncbi.nlm.nih.gov/entrez/

query.fcgi?cmd $=$ Retrieve\&db $=$ PubMed\&list_uids $=$

$14645018 \&$ dopt $=$ Abstract

Ricauda NA, Bo M, Molaschi M, et al. Home hospitalization service for acute uncomplicated first ischemic stroke in elderly patients: a randomized trial. J Am Geriatr Soc 2004:52:278-83.

http://www.ncbi.nlm.nih.gov/entrez/

query.fcgi? $\mathrm{cmd}=$ Retrieve $\& \mathrm{db}=$ pubmed \&dopt $=$ Abstract $\&$ list uids $=14728641$

Rice V, Stead L. Nursing interventions for smoking cessation. Cochrane Database Syst Rev 2004;(1):CD001188.

http://www.ncbi.nlm.nih.gov/entrez/

query.fcgi? cmd $=$ Retrieve \&db = PubMed\&list_uids =

14973964\&dopt $=$ Abstract

Rosenthal NR, Silverfield JC, Wu SC, et al. Tramadol/ Acetaminophen combination tablets for the treatment of pain associated with osteoarthritis flare in an elderly patient population. J Am Geriatr Soc 2004;52:374-80.

http://www.ncbi.nlm.nih.gov/entrez/

query.fcgi? $\mathrm{cmd}=$ Retrieve $\& \mathrm{db}=$ pubmed $\&$ dopt $=$ Abstract $\&$ list uids $=14962151$

Rostom A, Dube C, Jolicoeur E, et al. Gastroduodenal ulcers associated with the use of non-steroidal anti-inflammatory drugs: a systematic review of preventative pharmacological interventions. Ottawa: Canadian Coordinating Office for Health Technology Assessment (CCOHTA), Mar 2004.

http://www.ccohta.ca

Russell K, Wiebe N, Saenz A, et al. Glucocorticoids for croup. Cochrane Database Syst Rev 2004; (1):CD001955.

http://www.ncbi.nlm.nih.gov/entrez/

query.fcgi?cmd $=$ Retrieve $\&$ db $=$ PubMed\&list uids $=$

$14973975 \&$ dopt $=$ Abstract

Saunders D, Greig C, Young A, et al. Physical fitness training for stroke patients. Cochrane Database Syst Rev 2004;(1): CD003316.

http://www.ncbi.nlm.nih.gov/entrez/

query.fcgi?cmd $=$ Retrieve $\& \mathrm{db}=$ PubMed\&list_uids $=$

14974012\&dopt $=$ Abstract

Schmader KE, Hanlon JT, Pieper CF, et al. Effects of geriatric evaluation and management on adverse drug reactions and suboptimal prescribing in the frail elderly. Am $J$ Med 2004;116:394-401

http://www.ncbi.nlm.nih.gov/entrez/

query.fcgi? $\mathrm{cmd}=$ Retrieve $\& \mathrm{db}=$ pubmed $\& \mathrm{dopt}=$ Abstract $\&$ list_uids $=15006588$

Schneider SM, Prince-Paul M, Allen MJ, et al. Virtual reality as a distraction intervention for women receiving chemotherapy. Oncol Nurs Forum 2004;31:81-8.

http://www.ncbi.nlm.nih.gov/entrez/

query.fcgi?cmd $=$ Retrieve $\& \mathrm{db}=$ PubMed\&list_uids $=$

$14722591 \&$ dopt $=$ Abstract

Shea B, Wells G, Cranney A, et al. Calcium supplementation on bone loss in postmenopausal women. Cochrane Database Syst Rev 2004; ( 1):CD004526.

http://www.ncbi.nlm.nih.gov/entrez/

query.fcgi? $\mathrm{cmd}=$ Retrieve $\& \mathrm{db}=$ PubMed\&list_uids =

14974070\&dopt $=$ Abstract 
Shepperd S, Parkes J, McClaren J, et al. Discharge planning from hospital to home. Cochrane Database Syst Rev 2004; (1):CD000313.

http://www.ncbi.nlm.nih.gov/entrez/

query.fcgi?cmd $=$ Retrieve\&db $=$ PubMed\&list_uids $=$

14973952\&dopt $=$ Abstract

Smucny J, Flynn C, Becker L, et al. Beta ${ }_{2}$-agonists for acute bronchitis. Cochrane Database Syst Rev 2004;(1):CD001726.

http://www.ncbi.nlm.nih.gov/entrez/

query.fcgi?cmd $=$ Retrieve\&db $=$ PubMed\&list_uids $=$

14973971\&dopt $=$ Abstract

Soares-Weiser K, Goldberg E, Tamimi G, et al. Rotavirus vaccine for preventing diarrhoea. Cochrane Database Syst Rev 2004; ( 1):CD002848.

http://www.ncbi.nlm.nih.gov/entrez/

query.fcgi? $\mathrm{cmd}=$ Retrieve $\& \mathrm{db}=$ pubmed $\&$ dopt $=$ Abstract $\&$

list_uids $=14973994$

Soledad Cepeda M, Alvarez H, Morales O, et al. Addition of ultralow dose naloxone to postoperative morphine PCA: unchanged analgesia and opioid requirement but decreased incidence of opioid side effects. Pain 2004;107:41-6.

http://www.ncbi.nlm.nih.gov/entrez/

query.fcgi? $\mathrm{cmd}=$ Retrieve $\& \mathrm{db}=$ pubmed $\&$ dopt $=$ Abstract $\&$

list_uids $=14715387$

Stein MD, Solomon DA, Herman DS, et al. Pharmacotherapy plus psychotherapy for treatment of depression in active injection drug users. Arch Gen Psychiatry 2004;61:152-9.

http://www.ncbi.nlm.nih.gov/entrez/

query.fcgi? $\mathrm{cmd}=$ Retrieve $\& \mathrm{db}=$ pubmed $\&$ dopt $=$ Abstract $\&$

list uids $=14757591$

Steultjens E, Dekker J, Bouter L, et al. Occupational therapy for rheumatoid arthritis. Cochrane Database Syst Rev 2004; (1):CD003114.

http://www.ncbi.nlm.nih.gov/entrez/

query.fcgi?cmd $=$ Retrieve $\& \mathrm{db}=$ PubMed\&list_uids $=$

$14974005 \&$ dopt $=$ Abstract

Sur D, Gupta DN, Mondal SK, et al. Impact of zinc supplementation on diarrheal morbidity and growth pattern of low birth weight infants in Kolkata, India: a randomized, double-blind, placebo-controlled, community-based study. Pediatrics 2003;112:1327-32.

http://www.ncbi.nlm.nih.gov/entrez/

query.fcgi?cmd $=$ Retrieve $\& \mathrm{db}=$ PubMed\&list_uids $=$

$14654605 \& \mathrm{dopt}=$ Abstract

Sutton PM, Cresswell T, Livesey JP, et al. Treatment of anaemia after joint replacement. A double-blind, randomised, controlled trial of ferrous sulphate versus placebo. $J$ Bone Joint Surg Br 2004;86:31-3.

http://www.ncbi.nlm.nih.gov/entrez/

query.fcgi? $\mathrm{cmd}=$ Retrieve $\& \mathrm{db}=$ PubMed\&list uids $=$

14765861\&dopt $=$ Abstract

Temple J, Santy J. Pin site care for preventing infections associated with external bone fixators and pins. Cochrane Database Syst Rev 2004;(1):CD004551.

http://www.ncbi.nlm.nih.gov/entrez/

query.fcgi?cmd $=$ Retrieve \&db $=$ PubMed\&list_uids $=$

14974071\&dopt $=$ Abstract

Teunissen TA, de Jonge A, van Weel C, et al. Treating urinary incontinence in the elderly-conservative therapies that work: a systematic review. J Fam Pract 2004;53:25-30, 32. http://www.ncbi.nlm.nih.gov/entrez/

query.fcgi? $\mathrm{cmd}=$ Retrieve $\& \mathrm{db}=$ pubmed $\&$ dopt $=$ Abstract $\&$ list uids $=14709263$

Thomas A, Iacono D, Luciano AL, et al. Duration of amantadine benefit on dyskinesia of severe Parkinson's disease. J Neurol Neurosurg Psychiatry 2004;75:141-3.

http://www.ncbi.nlm.nih.gov/entrez/

query.fcgi? $\mathrm{cmd}=$ Retrieve $\& \mathrm{db}=$ pubmed \&dopt $=$ Abstract $\&$

list_uids $=14707325$

Tierney WM, Overhage JM, Murray MD, et al. Effects of computerized guidelines for managing heart disease in primary care. J Gen Intern Med 2003;18:967-76.

http://www.ncbi.nlm.nih.gov/entrez/

query.fcgi?cmd $=$ Retrieve $\& \mathrm{db}=$ PubMed\&list_uids = $14687254 \&$ dopt $=$ Abstract

van Zuuren E, Graber M, Hollis S, et al. Interventions for rosacea. Cochrane Database Syst Rev 2004;(1):CD003262.

http://www.ncbi.nlm.nih.gov/entrez/

query.fcgi?cmd $=$ Retrieve \&db $=$ PubMed\&list_uids $=$ 14974010\&dopt $=$ Abstract

Verhagen A, Scholten-Peeters G, de Bie R, et al. Conservative treatments for whiplash. Cochrane Database Syst Rev 2004; ( 1):CD003338.

http://www.ncbi.nlm.nih.gov/entrez/

query.fcgi? $\mathrm{cmd}=$ Retrieve $\& \mathrm{db}=$ PubMed\&list_uids $=$

14974013\&dopt $=$ Abstract

Vogel T, Verreault R, Gourdeau M, et al. Optimal duration of antibiotic therapy for uncomplicated urinary tract infection in older women: a double-blind randomized controlled trial. CMAJ 2004; 170:469-73.

http://www.ncbi.nlm.nih.gov/entrez/

query.fcgi? $\mathrm{cmd}=$ Retrieve $\& \mathrm{db}=$ pubmed $\&$ dopt $=$ Abstract $\&$ list_uids $=14970093$

Wallace S, Roe B, Williams $\mathrm{K}$, et al. Bladder training for urinary incontinence in adults. Cochrane Database Syst Rev 2004; (1):CD001308

http://www.ncbi.nlm.nih.gov/entrez/

query.fcgi?cmd $=$ Retrieve \&db = PubMed\&list_uids =

$14973967 \&$ dopt $=$ Abstract

Wegman A, van der Windt $\mathrm{D}$, van Tulder $\mathrm{M}$, et al. Nonsteroidal antiinflammatory drugs or acetaminophen for osteoarthritis of the hip or knee? A systematic review of evidence and guidelines. J Rheumatol 2004;31:344-54.

http://www.ncbi.nlm.nih.gov/entrez/

query.fcgi? $\mathrm{cmd}=$ Retrieve $\& \mathrm{db}=$ pubmed $\&$ dopt $=$ Abstract $\&$ list_uids $=14760807$

Wienecke T, Gøtzsche P. Paracetamol versus nonsteroidal anti-inflammatory drugs for rheumatoid arthritis. Cochrane Database Syst Rev 2004;(1):CD003789.

http://www.ncbi.nlm.nih.gov/entrez/

query.fcgi? $\mathrm{cmd}=$ Retrieve $\& \mathrm{db}=$ PubMed\&list_uids $=$ $14974037 \&$ dopt $=$ Abstract

Wilson AM, O'Byrne PM, Parameswaran K. Leukotriene receptor antagonists for allergic rhinitis: a systematic review and meta-analysis. Am J Med 2004;116:338-44.

http://www.ncbi.nlm.nih.gov/entrez/

query.fcgi? $\mathrm{cmd}=$ Retrieve $\& \mathrm{db}=$ pubmed $\& \mathrm{dopt}=$ Abstract $\&$ list_uids $=14984820$

Winkleby MA, Feighery E, Dunn M, et al. Effects of an advocacy intervention to reduce smoking among teenagers. Arch Pediatr Adolesc Med 2004;158:269-75. 
http://www.ncbi.nlm.nih.gov/entrez/ query.fcgi? $\mathrm{cmd}=$ Retrieve $\& \mathrm{db}=$ pubmed \&dopt $=$ Abstract $\&$ list uids $=14993087$

Yarbrough SS. Online exclusive: older women and breast cancer screening: research synthesis. Oncol Nurs Forum 2004;31:E9-E15

http://www.ncbi.nlm.nih.gov/entrez/

query.fcgi? $\mathrm{cmd}=$ Retrieve $\& \mathrm{db}=$ PubMed\&list_uids $=$ $14722601 \&$ dopt $=$ Abstract

Zobbe V, Gimbel H, Andersen BM, et al. Sexuality after total vs. subtotal hysterectomy. Acta Obstet Gynecol Scand 2004;83:191-6.

http://www.ncbi.nlm.nih.gov/entrez/

query.fcgi?cmd $=$ Retrieve $\& \mathrm{db}=$ pubmed $\&$ dopt $=$ Abstract $\&$

list_uids $=14756739$

\section{ASSESSMENT (SCREENING OR DIAGNOSIS)}

Anderson MR, Klink K, Cohrssen A. Evaluation of vaginal complaints. JAMA 2004;291:1368-79.

http://www.ncbi.nlm.nih.gov/entrez/

query.fcgi? $\mathrm{cmd}=$ Retrieve $\& \mathrm{db}=$ pubmed $\& \mathrm{dopt}=$ Abstract $\&$ list uids $=15026404$

Bachmann LM, Haberzeth S, Steurer J, et al. The accuracy of the Ottawa knee rule to rule out knee fractures: a systematic review. Ann Intern Med 2004;140:121-4.

http://www.ncbi.nlm.nih.gov/entrez/

query.fcgi? $\mathrm{cmd}=$ Retrieve $\& \mathrm{db}=$ pubmed $\&$ dopt $=$ Abstract $\&$ list_uids $=14734335$

Bordley WC, Viswanathan M, King VJ, et al. Diagnosis and testing in bronchiolitis: a systematic review. Arch Pediatr Adolesc Med 2004;158:119-26.

http://www.ncbi.nlm.nih.gov/entrez/

query.fcgi? $\mathrm{cmd}=$ Retrieve $\& \mathrm{db}=$ pubmed $\&$ dopt $=$ Abstract $\&$

list_uids $=14757603$

Stiell IG, Clement CM, McKnight RD, et al. The Canadian CSpine Rule versus the NEXUS Low-Risk Criteria in patients with trauma. $N$ Engl J Med 2003;349:2510-8.

http://www.ncbi.nlm.nih.gov/entrez/

query.fcgi?cmd $=$ Retrieve $\&$ db $=$ PubMed\&list_uids $=$

$14695411 \&$ dopt $=$ Abstract

Takata GS, Chan LS, Morphew T, et al. Evidence assessment of the accuracy of methods of diagnosing middle ear effusion in children with otitis media with effusion. Pediatrics 2003;112:1379-87.

http://www.ncbi.nlm.nih.gov/entrez/

query.fcgi?cmd $=$ Retrieve\&db $=$ PubMed\&list_uids $=$

14654613\&dopt $=$ Abstract

\section{PROGNOSIS}

Alarcón GS, Roseman JM, McGwin G Jr, et al. Systemic lupus erythematosus in three ethnic groups. XX. Damage as a predictor of further damage. Rheumatology (Oxford) 2004;43:202-5.

http://www.ncbi.nlm.nih.gov/entrez/

query.fcgi? $\mathrm{cmd}=$ Retrieve $\& \mathrm{db}=$ pubmed $\&$ dopt $=$ Abstract $\&$ list_uids $=12923289$

Doig CJ, Zygun DA, Fick GH, et al. Study of clinical course of organ dysfunction in intensive care. Crit Care Med 2004;32:384-90.

http://www.ncbi.nlm.nih.gov/entrez/

query.fcgi? $\mathrm{cmd}=$ Retrieve $\& \mathrm{db}=$ pubmed $\&$ dopt $=$ Abstract $\&$

list_uids $=14758152$
El-Serag HB, Talley NJ. The prevalence and clinical course of functional dyspepsia. Aliment Pharmacol Ther 2004;19:643-54. http://www.ncbi.nlm.nih.gov/entrez/

query.fcgi? $\mathrm{cmd}=$ Retrieve $\& \mathrm{db}=$ pubmed $\&$ dopt $=$ Abstract $\&$ list uids $=15023166$

Hoekstra RE, Ferrara TB, Couser RJ, et al. Survival and longterm neurodevelopmental outcome of extremely premature infants born at 23-26 weeks' gestational age at a tertiary center. Pediatrics 2004;113:el-6.

http://www.ncbi.nlm.nih.gov/entrez/

query.fcgi? $\mathrm{cmd}=$ Retrieve $\& \mathrm{db}=$ pubmed $\&$ dopt $=$ Abstract $\&$ list_uids $=14702487$

Kumral E, Oztürk O. Delusional state following acute stroke. Neurology 2004;62:110-3.

http://www.ncbi.nlm.nih.gov/entrez/

query.fcgi? $\mathrm{cmd}=$ Retrieve $\& \mathrm{db}=$ pubmed $\&$ dopt $=$ Abstract $\&$ list_uids $=14718709$

Marini C, Baldassarre M, Russo T, et al. Burden of first-ever ischemic stroke in the oldest old: evidence from a populationbased study. Neurology 2004;62:77-81.

http://www.ncbi.nlm.nih.gov/entrez/

query.fcgi? $\mathrm{cmd}=$ Retrieve $\& \mathrm{db}=$ pubmed $\&$ dopt $=$ Abstract $\&$ list_uids $=14718701$

Mercuri E, Barnett A, Rutherford M, et al. Neonatal cerebral infarction and neuromotor outcome at school age. Pediatrics 2004;113:95-100.

http://www.ncbi.nlm.nih.gov/entrez/

query.fcgi?cmd $=$ Retrieve $\& \mathrm{db}=$ pubmed $\&$ dopt $=$ Abstract $\&$ list_uids $=14702455$

Sommerfeld DK, Eek EU, Svensson AK, et al. Spasticity after stroke: its occurrence and association with motor impairments and activity limitations. Stroke 2004;35:134-9.

http://www.ncbi.nlm.nih.gov/entrez/

query.fcgi? $\mathrm{cmd}=$ Retrieve\&db $=$ PubMed\&list_uids $=$

$14684785 \& \mathrm{dopt}=$ Abstract

Sturm JW, Donnan GA, Dewey HM, et al. Determinants of handicap after stroke: the North East Melbourne Stroke Incidence Study (NEMESIS). Stroke 2004;35:715-20. http://www.ncbi.nlm.nih.gov/entrez/ query.fcgi? $\mathrm{cmd}=$ Retrieve $\& \mathrm{db}=$ pubmed $\&$ dopt $=$ Abstract $\&$ list_uids $=14963272$

Van Cleve L, Bossert E, Beecroft $\mathrm{P}$, et al. The pain experience of children with leukemia during the first year after diagnosis. Nurs Res 2004;53:1-10.

http://www.ncbi.nlm.nih.gov/entrez/

query.fcgi? $\mathrm{cmd}=$ Retrieve $\& \mathrm{db}=$ PubMed\&list uids $=$ 14726771\&dopt $=$ Abstract

\section{CAUSATION}

Albertsen K, Andersen AM, Olsen J, et al. Alcohol consumption during pregnancy and the risk of preterm delivery. Am J Epidemiol 2004;159:155-61.

http://www.ncbi.nlm.nih.gov/entrez/

query.fcgi?cmd $=$ Retrieve $\& \mathrm{db}=$ PubMed\&list_uids $=$ $14718217 \&$ dopt $=$ Abstract

Araki A, Murotani Y, Kamimiya F, et al. Low well-being is an independent predictor for stroke in elderly patients with diabetes mellitus. J Am Geriatr Soc 2004;52:205-10.

http://www.ncbi.nlm.nih.gov/entrez/

query.fcgi? $\mathrm{cmd}=$ Retrieve $\& \mathrm{db}=$ pubmed $\&$ dopt $=$ Abstract $\&$ list_uids $=14728628$ 
Beebe-Dimmer J, Lynch JW, Turrell G, et al. Childhood and adult socioeconomic conditions and 31-year mortality risk in women. Am J Epidemiol 2004;159:481-90.

http://www.ncbi.nlm.nih.gov/entrez/

query.fcgi? $\mathrm{cmd}=$ Retrieve $\& \mathrm{db}=$ pubmed $\&$ dopt $=$ Abstract $\&$ list_uids $=14977644$

Diehr P, Beresford SA. The relation of dietary patterns to future survival, health, and cardiovascular events in older adults. J Clin Epidemiol 2003;56:1224-35.

http://www.ncbi.nlm.nih.gov/entrez/

query.fcgi?cmd $=$ Retrieve\&db $=$ PubMed\&list_uids $=$ $14680674 \&$ dopt $=$ Abstract

Etminan M, Hemmelgarn B, Delaney JA, et al. Use of lithium and the risk of injurious motor vehicle crash in elderly adults: case-control study nested within a cohort. $B M J$ 2004;328:558-9.

http://www.ncbi.nlm.nih.gov/entrez/

query.fcgi? $\mathrm{cmd}=$ Retrieve $\& \mathrm{db}=$ pubmed \&dopt $=$ Abstract $\&$ list_uids $=14732760$

Goodman JH. Paternal postpartum depression, its relationship to maternal postpartum depression, and implications for family health. $J$ Adv Nurs 2004;45:26-35.

http://www.ncbi.nlm.nih.gov/entrez/

query.fcgi?cmd $=$ Retrieve $\& \mathrm{db}=$ PubMed\&list_uids $=$

14675298\&dopt $=$ Abstract

Hillis SD, Anda RF, Dube SR, et al. The association between adverse childhood experiences and adolescent pregnancy, long-term psychosocial consequences, and fetal death. Pediatrics 2004;113:320-7.

http://www.ncbi.nlm.nih.gov/entrez/

query.fcgi? $\mathrm{cmd}=$ Retrieve $\& \mathrm{db}=$ pubmed $\&$ dopt $=$ Abstract $\&$

list_uids $=14754944$

Howard AA, Arnsten JH, Gourevitch MN. Effect of alcohol consumption on diabetes mellitus: a systematic review. Ann Intern Med 2004;140:211-9.

http://www.ncbi.nlm.nih.gov/entrez/

query.fcgi? $\mathrm{cmd}=$ Retrieve $\& \mathrm{db}=$ pubmed\&dopt $=$ Abstract $\&$

list_uids $=14757619$

Juon HS, Ensminger ME, Feehan M. Childhood adversity and later mortality in an urban African American cohort. Am J Public Health 2003;93:2044-6.

http://www.ncbi.nlm.nih.gov/entrez/

query.fcgi? $\mathrm{cmd}=$ Retrieve $\& \mathrm{db}=$ PubMed\&list_uids $=$

14652330\&dopt $=$ Abstract

Kanaya AM, Vittinghoff E, Shlipak MG, et al. Association of total and central obesity with mortality in postmenopausal women with coronary heart disease. Am J Epidemiol 2003; 158:1161-70.

http://www.ncbi.nlm.nih.gov/entrez/

query.fcgi? $\mathrm{cmd}=$ Retrieve $\& \mathrm{db}=$ PubMed\&list_uids $=$

$14652301 \& d o p t=$ Abstract

Lamarca R, Ferrer M, Andersen PK, et al. A changing relationship between disability and survival in the elderly population: differences by age. J Clin Epidemiol 2003;56:1192201.

http://www.ncbi.nlm.nih.gov/entrez/

query.fcgi? $\mathrm{cmd}=$ Retrieve $\& \mathrm{db}=$ PubMed\&list_uids $=$ 14680670\&dopt $=$ Abstract

Rubinson L, Diette GB, Song X, et al. Low caloric intake is associated with nosocomial bloodstream infections in patients in the medical intensive care unit. Crit Care Med 2004;32:350-7. http://www.ncbi.nlm.nih.gov/entrez/ query.fcgi? $\mathrm{cmd}=$ Retrieve $\& \mathrm{db}=$ pubmed $\&$ dopt $=$ Abstract $\&$ list_uids $=14758147$

Sin DD, Spier S, Svenson LW, et al. The relationship between birth weight and childhood asthma: a population-based cohort study. Arch Pediatr Adolesc Med 2004;158:60-4.

http://www.ncbi.nlm.nih.gov/entrez/

query.fcgi?cmd $=$ Retrieve $\& \mathrm{db}=$ PubMed\&list_uids $=$ 14706960\&dopt $=$ Abstract

Sollid CP, Wisborg K, Hjort J, et al. Eating disorder that was diagnosed before pregnancy and pregnancy outcome. Am J Obstet Gynecol 2004; 190:206-10.

http://www.ncbi.nlm.nih.gov/entrez/

query.fcgi?cmd $=$ Retrieve $\& \mathrm{db}=$ PubMed\&list_uids $=$

$14749661 \&$ dopt $=$ Abstract

Surkan PJ, Stephansson O, Dickman PW, et al. Previous preterm and small-for-gestational-age births and the subsequent risk of stillbirth. $N$ Engl J Med 2004;350:777-85.

http://www.ncbi.nlm.nih.gov/entrez/

query.fcgi? $\mathrm{cmd}=$ Retrieve $\& \mathrm{db}=$ pubmed $\&$ dopt $=$ Abstract $\&$

list_uids $=14973215$

Tuomilehto J, Hu G, Bidel S, et al. Coffee consumption and risk of type 2 diabetes mellitus among middle-aged Finnish men and women. JAMA 2004;291:1213-9.

http://www.ncbi.nlm.nih.gov/entrez/

query.fcgi? $\mathrm{cmd}=$ Retrieve $\& \mathrm{db}=$ pubmed $\&$ dopt $=$ Abstract $\&$ list uids $=15010442$

Weiss JL, Malone FD, Vidaver J, et al. Threatened abortion: a risk factor for poor pregnancy outcome, a population-based screening study. Am J Obstet Gynecol 2004;190:745-50. http://www.ncbi.nlm.nih.gov/entrez/ query.fcgi? $\mathrm{cmd}=$ Retrieve $\& \mathrm{db}=$ pubmed $\&$ dopt $=$ Abstract $\&$ list_uids $=15042008$

Wheeler PG, Bresnahan K, Shephard BA, et al. Short stature and functional impairment: a systematic review. Arch Pediatr Adolesc Med 2004;158:236-43.

http://www.ncbi.nlm.nih.gov/entrez/

query.fcgi? $\mathrm{cmd}=$ Retrieve $\& \mathrm{db}=$ pubmed $\&$ dopt $=$ Abstract $\&$ list_uids $=14993082$

\section{ECONOMICS}

Angus DC, Clermont G, Watson RS, et al. Cost-effectiveness of inhaled nitric oxide in the treatment of neonatal respiratory failure in the United States. Pediatrics 2003;112:1351-60. http://www.ncbi.nlm.nih.gov/entrez/ query.fcgi?cmd $=$ Retrieve\&db $=$ PubMed\&list_uids $=$ $14654609 \&$ dopt $=$ Abstract

Kekki M, Kurki T, Kotomäki T, et al. Cost-effectiveness of screening and treatment for bacterial vaginosis in early pregnancy among women at low risk for preterm birth. Acta Obstet Gynecol Scand 2004;83:27-36.

http://www.ncbi.nlm.nih.gov/entrez/

query.fcgi? $\mathrm{cmd}=$ Retrieve $\& \mathrm{db}=$ PubMed\&list_uids $=$ $14678083 \&$ dopt $=$ Abstract

Patel A, Knapp M, Perez I, et al. Alternative strategies for stroke care: cost-effectiveness and cost-utility analyses from a prospective randomized controlled trial. Stroke 2004;35:196203.

http://www.ncbi.nlm.nih.gov/entrez/

query.fcgi? $\mathrm{cmd}=$ Retrieve $\& \mathrm{db}=$ PubMed\&list_uids $=$ 14684783\&dopt $=$ Abstract 
10 of 13

Renehan AG, O'Dwyer ST, Whynes DK. Cost effectiveness analysis of intensive versus conventional follow up after curative resection for colorectal cancer. BMJ 2004;328:81-5. http://www.ncbi.nlm.nih.gov/entrez/ query.fcgi?cmd $=$ Retrieve $\& \mathrm{db}=$ PubMed\&list_uids $=$ $14715603 \& d o p t=$ Abstract

Sculpher M, Manca A, Abbott J, et al. Cost effectiveness analysis of laparoscopic hysterectomy compared with standard hysterectomy: results from a randomised trial. BMJ 2004;328:134-9.

http://www.ncbi.nlm.nih.gov/entrez/ query.fcgi?cmd $=$ Retrieve $\& \mathrm{db}=$ PubMed\&list uids $=$ 14711748\&dopt $=$ Abstract

\section{QUALITY IMPROVEMENT}

Gibson PG, Powell H. Written action plans for asthma: an evidence-based review of the key components. Thorax 2004;59:94-9.

http://www.ncbi.nlm.nih.gov/entrez/ query.fcgi?cmd $=$ Retrieve $\& \mathrm{db}=$ pubmed $\& \mathrm{dopt}=$ Abstract $\&$ list uids $=14760143$

Margolis PA, Lannon CM, Stuart JM, et al. Practice based education to improve delivery systems for prevention in primary care: randomised trial. BMJ 2004;328:388.

http://www.ncbi.nlm.nih.gov/entrez/

query.fcgi?cmd $=$ Retrieve\&db $=$ PubMed\&list_uids $=$

14766718\&dopt $=$ Abstract

\section{CLINICAL PREDICTION GUIDE}

Balestreri M, Czosnyka M, Chatfield DA, et al. Predictive value of Glasgow Coma Scale after brain trauma: change in trend over the past ten years. J Neurol Neurosurg Psychiatry 2004;75:161-2.

http://www.ncbi.nlm.nih.gov/entrez/

query.fcgi?cmd $=$ Retrieve $\& \mathrm{db}=$ pubmed $\&$ dopt $=$ Abstract $\&$ list uids $=14077332$

Brophy JM, Dagenais GR, McSherry F, et al. A multivariate model for predicting mortality in patients with heart failure and systolic dysfunction. Am J Med 2004;116:300-4.

http://www.ncbi.nlm.nih.gov/entrez/

query.fcgi? $\mathrm{cmd}=$ Retrieve $\& \mathrm{db}=$ pubmed $\&$ dopt $=$ Abstract $\&$ list_uids $=14984814$

Celli BR, Cote CG, Marin JM, et al. The body-mass index, airflow obstruction, dyspnea, and exercise capacity index in chronic obstructive pulmonary disease. $N$ Engl $J$ Med 2004;350:1005-12.

http://www.ncbi.nlm.nih.gov/entrez/

query.fcgi? $\mathrm{cmd}=$ Retrieve $\& \mathrm{db}=$ pubmed\&dopt $=$ Abstract $\&$ list_uids = 14999112

Counsell C, Dennis M, McDowall M. Predicting functional outcome in acute stroke: comparison of a simple six variable model with other predictive systems and informal clinical prediction. J Neurol Neurosurg Psychiatry 2004;75:401-5. http://www.ncbi.nlm.nih.gov/entrez/

query.fcgi?cmd $=$ Retrieve $\& \mathrm{db}=$ pubmed $\&$ dopt $=$ Abstract $\&$ list uids $=14966155$

Dendukuri N, McCusker J, Belzile E. The Identification of Seniors at Risk screening tool: further evidence of concurrent and predictive validity. J Am Geriatr Soc 2004;52:290-6. http://www.ncbi.nlm.nih.gov/entrez/ query.fcgi?cmd $=$ Retrieve $\& \mathrm{db}=$ pubmed $\&$ dopt $=$ Abstract $\&$ list_uids $=14728643$
Hak E, Wei F, Nordin J, et al. Development and validation of a clinical prediction rule for hospitalization due to pneumonia or influenza or death during influenza epidemics among community-dwelling elderly persons. J Infect Dis 2004; 189: $450-8$.

http://www.ncbi.nlm.nih.gov/entrez/ query.fcgi? $\mathrm{cmd}=$ Retrieve $\& \mathrm{db}=$ pubmed $\&$ dopt $=$ Abstract $\&$ list uids $=14745702$

Hashima JN, Eden KB, Osterweil P, et al. Predicting vaginal birth after cesarean delivery: a review of prognostic factors and screening tools. Am J Obstet Gynecol 2004;190:547-55. http://www.ncbi.nlm.nih.gov/entrez/

query.fcgi? $\mathrm{cmd}=$ Retrieve $\& \mathrm{db}=$ pubmed $\&$ dopt $=$ Abstract $\&$ list uids $=14981405$

Tay SY, Sloan EP, Zun L, et al. Comparison of the New Injury Severity Score and the Injury Severity Score. J Trauma 2004;56:162-4

http://www.ncbi.nlm.nih.gov/entrez/

query.fcgi?cmd $=$ Retrieve\&db $=$ PubMed\&list_uids $=$

$14749583 \&$ dopt $=$ Abstract

Predicting outcome after acute ischemic stroke: an external validation of prognostic models. German Stroke Study Collaboration. Neurology 2004;62:581-5.

http://www.ncbi.nlm.nih.gov/entrez/

query.fcgi? $\mathrm{cmd}=$ Retrieve $\& \mathrm{db}=$ pubmed $\&$ dopt $=$ Abstract $\&$ list_uids $=14981174$

\section{QUALITATIVE}

Beck CT. Birth trauma: in the eye of the beholder. Nurs Res 2004;53:28-35

http://www.ncbi.nlm.nih.gov/entrez/

query.fcgi? $\mathrm{cmd}=$ Retrieve $\& \mathrm{db}=$ PubMed\&list_uids $=$

$14726774 \&$ dopt $=$ Abstract

Bennstam AL, Strandmark M, Diwan VK. Perception of tuberculosis in the Democratic Republic of Congo: wali ya nkumu in the Mai Ndombe district. Qual Health Res 2004; 14:299-312.

http://www.ncbi.nlm.nih.gov/entrez/

query.fcgi?cmd $=$ Retrieve $\& \mathrm{db}=$ pubmed $\&$ dopt $=$ Abstract $\&$ list_uids $=15011899$

Bissell P, May CR, Noyce PR. From compliance to concordance: barriers to accomplishing a re-framed model of health care interactions. Soc Sci Med 2004;58:851-62. http://www.ncbi.nlm.nih.gov/entrez/ query.fcgi? $\mathrm{cmd}=$ Retrieve $\& \mathrm{db}=$ pubmed $\&$ dopt $=$ Abstract $\&$ list_uids $=14672598$

Bonner A, Walker A. Nephrology nursing: blurring the boundaries: the reality of expert practice. $J$ Clin Nurs 2004; 13:210-8

http://www.ncbi.nlm.nih.gov/entrez/

query.fcgi?cmd $=$ Retrieve $\& \mathrm{db}=$ PubMed\&list_uids =

14723673\&dopt $=$ Abstract

Bourjolly JN, Hirschman KB, Zieber NA. The impact of managed health care in the United States on women with breast cancer and the providers who treat them. Cancer Nurs 2004:27:45-54.

Bulsara C, Ward A, Joske D. Haematological cancer patients: achieving a sense of empowerment by use of strategies to control illness. J Clin Nurs 2004;13:251-8.

http://www.ncbi.nlm.nih.gov/entrez/

query.fcgi? $\mathrm{cmd}=$ Retrieve $\& \mathrm{db}=$ PubMed\&list uids $=$ $14723678 \& d o p t=$ Abstract 
Chan S, Levy V. Postnatal depression: a qualitative study of the experiences of a group of Hong Kong Chinese women. $J$ Clin Nurs 2004;13:120-3.

http://www.ncbi.nlm.nih.gov/entrez/

query.fcgi?cmd $=$ Retrieve \&db $=$ PubMed\&list_uids $=$

$14687304 \&$ dopt $=$ Abstract

Collopy KS. "I couldn't think that far": infertile women's decision making about multifetal reduction. Res Nurs Health 2004;27:75-86.

http://www.ncbi.nlm.nih.gov/entrez/

query.fcgi? $\mathrm{cmd}=$ Retrieve $\& \mathrm{db}=$ pubmed $\&$ dopt $=$ Abstract $\&$ list_uids $=15042634$

DelVecchio Good MJ, Gadmer NM, Ruopp P, et al. Narrative nuances on good and bad deaths: internists' tales from hightechnology work places. Soc Sci Med 2004;58:939-53.

http://www.ncbi.nlm.nih.gov/entrez/

query.fcgi? $\mathrm{cmd}=$ Retrieve $\& \mathrm{db}=$ pubmed $\&$ dopt $=$ Abstract $\&$ list_uids $=14732607$

Dinos S, Stevens S, Serfaty M, et al. Stigma: the feelings and experiences of 46 people with mental illness. Qualitative study. Br J Psychiatry 2004;184:176-81.

http://www.ncbi.nlm.nih.gov/entrez/

query.fcgi? $\mathrm{cmd}=$ Retrieve $\& \mathrm{db}=$ pubmed $\&$ dopt $=$ Abstract $\&$ list_uids $=14754832$

Farran CJ, Loukissa D, Perraud S, et al. Alzheimer's disease caregiving information and skills. Part II: family caregiver issues and concerns. Res Nurs Health 2004;27:40-51.

http://www.ncbi.nlm.nih.gov/entrez/

query.fcgi?cmd $=$ Retrieve $\& \mathrm{db}=$ PubMed\&list_uids $=$

$14745855 \&$ dopt $=$ Abstract

Farrell M, Holmes G, Coldicutt P, et al. Management of childhood constipation: parents' experiences. J Adv Nurs 2003;44:479-89.

http://www.ncbi.nlm.nih.gov/entrez/

query.fcgi?cmd $=$ Retrieve\&db $=$ PubMed\&list_uids $=$

14651696\&dopt $=$ Abstract

Fletcher CE. Health care provider's perceptions of spirituality while caring for veterans. Qual Health Res 2004;14:546-61.

http://www.ncbi.nlm.nih.gov/entrez/

query.fcgi?cmd $=$ Retrieve $\& \mathrm{db}=$ pubmed $\&$ dopt $=$ Abstract $\&$ list_uids $=15068579$

Gramling L, Hickman K, Bennett S. What makes a good family-centered partnership between women and their practitioners? A qualitative study. Birth 2004;31:43-8. http://www.ncbi.nlm.nih.gov/entrez/ query.fcgi? $\mathrm{cmd}=$ Retrieve $\& \mathrm{db}=$ pubmed $\& \mathrm{dopt}=$ Abstract $\&$ list_uids = 15015992

Green SE. "What do you mean 'what's wrong with her?" stigma and the lives of families of children with disabilities. Soc Sci Med 2003;57:1361-74.

http://www.ncbi.nlm.nih.gov/entrez/

query.fcgi? $\mathrm{cmd}=$ Retrieve\&db $=$ PubMed\&list_uids $=$

12927467\&dopt $=$ Abstract

Haugli L, Strand E, Finset A. How do patients with rheumatic disease experience their relationship with their doctors? A qualitative study of experiences of stress and support in the doctor-patient relationship. Patient Educ Counsel 2004;52:169174.

Helseth S, Ulfsaet N. Having a parent with cancer: coping and quality of life of children during serious illness in the family. Cancer Nurs 2003;26:355-62. http://www.ncbi.nlm.nih.gov/entrez/ query.fcgi?cmd $=$ Retrieve $\& \mathrm{db}=$ PubMed\&list_uids = $14710796 \&$ dopt $=$ Abstract

Heneghan AM, Mercer M, DeLeone NL. Will mothers discuss parenting stress and depressive symptoms with their child's pediatrician? Pediatrics 2004;113:460-7. http://www.ncbi.nlm.nih.gov/entrez/

query.fcgi? $\mathrm{cmd}=$ Retrieve $\& \mathrm{db}=$ pubmed $\&$ dopt $=$ Abstract $\&$ list_uids = 14993535

Heyman B, Swain J, Gillman M. Organisational simplification and secondary complexity in health services for adults with learning disabilities. Soc Sci Med 2004;58:357-67.

http://www.ncbi.nlm.nih.gov/entrez/

query.fcgi? $\mathrm{cmd}=$ Retrieve $\& \mathrm{db}=$ PubMed\&list_uids $=$ $14604621 \&$ dopt $=$ Abstract

Honey A. Benefits and drawbacks of employment: perspectives of people with mental illness. Qual Health Res 2004: 14:381-95.

http://www.ncbi.nlm.nih.gov/entrez/

query.fcgi? $\mathrm{cmd}=$ Retrieve $\& \mathrm{db}=$ pubmed \&dopt $=$ Abstract $\&$ list_uids $=15011905$

Husain-Gambles M, Neal RD, Dempsey O, et al. Missed appointments in primary care: questionnaire and focus group study of health professionals. Br J Gen Pract 2004;54:108-13. http://www.ncbi.nlm.nih.gov/entrez/

query.fcgi? $\mathrm{cmd}=$ Retrieve $\& \mathrm{db}=$ pubmed $\&$ dopt $=$ Abstract $\&$ list_uids $=14965389$

Iezzoni LI, O'Day BL, Killeen M, et al. Communicating about health care: observations from persons who are deaf or hard of hearing. Ann Intern Med 2004;140:356-62.

http://www.ncbi.nlm.nih.gov/entrez/

query.fcgi? $\mathrm{cmd}=$ Retrieve $\& \mathrm{db}=$ pubmed $\&$ dopt $=$ Abstract $\&$ list_uids $=14996677$

Im EO, Choe MA. Korean women's attitudes toward physical activity. Res Nurs Health 2004;27:4-18.

http://www.ncbi.nlm.nih.gov/entrez/

query.fcgi? $\mathrm{cmd}=$ Retrieve $\& \mathrm{db}=$ PubMed\&list_uids $=$

14745852\&dopt $=$ Abstract

Johnson G, Helman C. Remedy or cure? Lay beliefs about over-the-counter medicines for coughs and colds. Br J Gen Pract 2004;54:98-102.

http://www.ncbi.nlm.nih.gov/entrez/

query.fcgi? $\mathrm{cmd}=$ Retrieve $\& \mathrm{db}=$ pubmed $\&$ dopt $=$ Abstract $\&$ list_uids $=14965387$

Kälvemark S, Höglund AT, Hansson MG, et al. Living with conflicts-ethical dilemmas and moral distress in the health care system. Soc Sci Med 2004;58:1075-84.

http://www.ncbi.nlm.nih.gov/entrez/

query.fcgi? $\mathrm{cmd}=$ Retrieve $\& \mathrm{db}=$ pubmed $\&$ dopt $=$ Abstract $\&$ list_uids $=14723903$

Karhila P, Kettunen T, Poskiparta M, et al. Negotiation in type 2 diabetes counseling: from problem recognition to mutual acceptance during lifestyle counseling. Qual Health Res 2003; 13:1205-24.

http://www.ncbi.nlm.nih.gov/entrez/ query.fcgi?cmd $=$ Retrieve $\& \mathrm{db}=$ PubMed\&list_uids = 14606410\&dopt $=$ Abstract

Kiata L, Kerse N. Intercultural residential care in New Zealand. Qual Health Res 2004;14:313-27.

http://www.ncbi.nlm.nih.gov/entrez/

query.fcgi? $\mathrm{cmd}=$ Retrieve $\& \mathrm{db}=$ pubmed $\&$ dopt $=$ Abstract $\&$ list_uids $=15011900$ 
12 of 13

King M, Smith G, Bartlett A. Treatments of homosexuality in Britain since the 1950s-an oral history: the experience of professionals. BMJ 2004;328:429.

http://www.ncbi.nlm.nih.gov/entrez/

query.fcgi? $\mathrm{cmd}=$ Retrieve $\& \mathrm{db}=$ PubMed\&list_uids $=$

14751921\&dopt $=$ Abstract

Lewis DK, Robinson J, Wilkinson E. Factors involved in deciding to start preventive treatment: qualitative study of clinicians' and lay people's attitudes. BMJ 2003;327:841-6. http://www.ncbi.nlm.nih.gov/entrez/

query.fcgi?cmd $=$ Retrieve \&db $=$ PubMed\&list_uids $=$ 14551099\&dopt $=$ Abstract

Long SO. Cultural scripts for a good death in Japan and the United States: similarities and differences. Soc Sci Med 2004;58:913-28.

http://www.ncbi.nlm.nih.gov/entrez/

query.fcgi? $\mathrm{cmd}=$ Retrieve $\& \mathrm{db}=$ pubmed $\&$ dopt $=$ Abstract $\&$ list_uids $=14732605$

Luk WS. The HRQoL of renal transplant patients. J Clin Nurs 2004; 13:201-9.

http://www.ncbi.nlm.nih.gov/entrez/

query.fcgi? $\mathrm{cmd}=$ Retrieve $\& \mathrm{db}=$ PubMed\&list_uids $=$

$14723672 \&$ dopt $=$ Abstract

Maijala H, Paavilainen E, Väisänen L, et al. Caregivers' experiences of interaction with families expecting a fetally impaired child. $J$ Clin Nurs 2004;13:376-85.

http://www.ncbi.nlm.nih.gov/entrez/

query.fcgi? $\mathrm{cmd}=$ Retrieve $\& \mathrm{db}=$ pubmed $\&$ dopt $=$ Abstract $\&$

list_uids $=15009340$

Maliski SL, Clerkin B, Letwin MS. Describing a nurse case manager intervention to empower low-income men with prostate cancer. Oncol Nurs Forum 2004;31:57-64.

http://www.ncbi.nlm.nih.gov/entrez/

query.fcgi?cmd $=$ Retrieve $\& \mathrm{db}=$ PubMed\&list_uids $=$

14722588\&dopt $=$ Abstract

McNeill T. Fathers' experience of parenting a child with juvenile rheumatoid arthritis. Qual Health Res 2004; 14:526-45. http://www.ncbi.nlm.nih.gov/entrez/

query.fcgi? $\mathrm{cmd}=$ Retrieve $\& \mathrm{db}=$ pubmed $\&$ dopt $=$ Abstract $\&$ list_uids $=15068578$

Mok E, Martinson I, Wong TKS. Individual empowerment among Chinese cancer patients in Hong Kong. West J Nurs Res 2004;26:59-75.

http://www.ncbi.nlm.nih.gov/entrez/

query.fcgi? $\mathrm{cmd}=$ Retrieve $\& \mathrm{db}=$ pubmed $\&$ dopt $=$ Abstract $\&$

list_uids $=14984648$

Montbriand MJ. Seniors' survival trajectories and the illness connection. Qual Health Res 2004;14:449-61.

http://www.ncbi.nlm.nih.gov/entrez/

query.fcgi? $\mathrm{cmd}=$ Retrieve $\& \mathrm{db}=$ pubmed $\& \mathrm{dopt}=$ Abstract $\&$

list_uids $=15068573$

Morrison L. Traditions in transition: young people's risk for HIV in Chiang Mai, Thailand. Qual Health Res 2004;14:328-44. http://www.ncbi.nlm.nih.gov/entrez/

query.fcgi? $\mathrm{cmd}=$ Retrieve $\& \mathrm{db}=$ pubmed $\&$ dopt $=$ Abstract $\&$ list_uids = 15011901

Navon L, Morag A. Advanced prostate cancer patients' ways of coping with the hormonal therapy's effect on body, sexuality, and spousal ties. Qual Health Res 2003;13:1378-92. http://www.ncbi.nlm.nih.gov/entrez/ query.fcgi?cmd $=$ Retrieve $\& \mathrm{db}=$ PubMed\&list_uids $=$ 14658352\&dopt $=$ Abstract
Nordgren L, Olsson H. Palliative care in a coronary care unit: a qualitative study of physicians' and nurses' perceptions. $J$ Clin Nurs 2004;13:185-93.

http://www.ncbi.nlm.nih.gov/entrez/

query.fcgi?cmd $=$ Retrieve \&db $=$ PubMed\&list_uids $=$

$1472367 \& d o p t=$ Abstract

Oates MR, Cox JL, Neema S, et al. Postnatal depression across countries and cultures: a qualitative study. $\mathrm{Br} J$ Psychiatry Suppl 2004;46:s10-6.

http://www.ncbi.nlm.nih.gov/entrez/

query.fcgi? $\mathrm{cmd}=$ Retrieve $\& \mathrm{db}=$ pubmed $\&$ dopt $=$ Abstract $\&$ list uids $=14754813$

Palfreyman SJ, Drewery-Carter K, Rigby K, et al. Varicose veins: a qualitative study to explore expectations and reasons for seeking treatment. J Clin Nurs 2004;13:332-40.

http://www.ncbi.nlm.nih.gov/entrez/

query.fcgi? $\mathrm{cmd}=$ Retrieve $\& \mathrm{db}=$ pubmed $\&$ dopt $=$ Abstract $\&$ list_uids $=15009336$

Peckover S. Health visitors' understandings of domestic violence. J Adv Nurs 2003;44:200-8.

http://www.ncbi.nlm.nih.gov/entrez/

query.fcgi?cmd $=$ Retrieve $\& \mathrm{db}=$ PubMed\&list_uids $=$

14521686\&dopt $=$ Abstract

Persoon A, Heinen MM, van der Vleuten CJ, et al. Leg ulcers: a review of their impact on daily life. J Clin Nurs 2004;13:34154.

http://www.ncbi.nlm.nih.gov/entrez/

query.fcgi?cmd $=$ Retrieve $\& \mathrm{db}=$ pubmed $\&$ dopt $=$ Abstract $\&$ list uids $=15009337$

Poindexter CC. Medical profiling: narratives of privilege, prejudice, and HIV stigma. Qual Health Res 2004;14:496-512. http://www.ncbi.nlm.nih.gov/entrez/

query.fcgi? $\mathrm{cmd}=$ Retrieve $\& \mathrm{db}=$ pubmed $\&$ dopt $=$ Abstract $\&$ list_uids $=15068576$

Radina ME, Armer JM, Culbertson SD, et al. Post-breast cancer lymphedema: understanding women's knowledge of their condition. Oncol Nurs Forum 2004;31:97-104.

http://www.ncbi.nlm.nih.gov/entrez/

query.fcgi?cmd $=$ Retrieve $\&$ db $=$ PubMed\&list_uids $=$

$14722593 \&$ dopt $=$ Abstract

Randers I, Mattiasson AC. Autonomy and integrity: upholding older adult patients' dignity. $J$ Adv Nurs 2004;45:63-71. http://www.ncbi.nlm.nih.gov/entrez/

query.fcgi? $\mathrm{cmd}=$ Retrieve $\& \mathrm{db}=$ PubMed\&list_uids $=$

14675302\&dopt $=$ Abstract

Reynolds F, Prior S. "Sticking jewels in your life": exploring women's strategies for negotiating an acceptable quality of life with multiple sclerosis. Qual Health Res 2003;13:1225-51. http://www.ncbi.nlm.nih.gov/entrez/

query.fcgi?cmd $=$ Retrieve\&db $=$ PubMed\&list_uids $=$

14606411\&dopt $=$ Abstract

Salazar MK, Napolitano M, Scherer JA, et al. Hispanic adolescent farmworkers' perceptions associated with pesticide exposure. West J Nurs Res 2004;26:146-66.

http://www.ncbi.nlm.nih.gov/entrez/

query.fcgi? $\mathrm{cmd}=$ Retrieve $\& \mathrm{db}=$ pubmed $\&$ dopt $=$ Abstract $\&$ list_uids $=15005979$

Schneider B, Scissons H, Arney L, et al. Communication between people with schizophrenia and their medical professionals: a participatory research project. Qual Health Res 2004; 14:562-77. 
http://www.ncbi.nlm.nih.gov/entrez/

query.fcgi? $\mathrm{cmd}=$ Retrieve $\& \mathrm{db}=$ pubmed $\& \mathrm{dopt}=$ Abstract $\&$ list_uids $=15068580$

Sheehan K, Kim LE, Galvin JP Jr. Urban children's perceptions of violence. Arch Pediatr Adolesc Med 2004;158:74-7. http://www.ncbi.nlm.nih.gov/entrez/ query.fcgi?cmd $=$ Retrieve \&db $=$ PubMed\&list_uids $=$ 14706962\&dopt $=$ Abstract

Skea Z, Harry V, Bhattacharya S, et al. Women's perceptions of decision-making about hysterectomy. BJOG 2004;111:13342.

http://www.ncbi.nlm.nih.gov/entrez/

query.fcgi?cmd $=$ Retrieve $\& \mathrm{db}=$ pubmed $\&$ dopt $=$ Abstract $\&$

list_uids $=14723750$

Smith G, Bartlett A, King M. Treatments of homosexuality in Britain since the 1950s-an oral history: the experience of patients. BMJ 2004;328:427.

http://www.ncbi.nlm.nih.gov/entrez/

query.fcgi?cmd $=$ Retrieve $\& \mathrm{db}=$ PubMed\&list_uids $=$

1475192\&dopt $=$ Abstract

Sullivan T, Weinert C, Cudney S. Management of chronic illness: voices of rural women. J Adv Nurs 2003;44:566-74.

http://www.ncbi.nlm.nih.gov/entrez/

query.fcgi?cmd $=$ Retrieve \&db $=$ PubMed\&list_uids $=$

14651679\&dopt $=$ Abstract

Tishelman C, Bernhardson BM, Blomberg K, et al. Complexity in caring for patients with advanced cancer. $J$ Adv Nurs 2004;45:420-9.

http://www.ncbi.nlm.nih.gov/entrez/

query.fcgi?cmd $=$ Retrieve $\& \mathrm{db}=$ PubMed\&list_uids $=$

14756836\&dopt $=$ Abstract

Trovo de Araujo MM, Paes da Silva MJ. Communication with dying patients-perception of intensive care units nurses in Brazil. J Clin Nurs 2004;13:143-9. http://www.ncbi.nlm.nih.gov/entrez/ query.fcgi? $\mathrm{cmd}=$ Retrieve $\& \mathrm{db}=$ PubMed\&list_uids $=$ $14723665 \&$ dopt $=$ Abstract

Townsend A, Hunt K, Wyke S. Managing multiple morbidity in mid-life: a qualitative study of attitudes to drug use. BMJ 2003;327:837-42.

http://www.ncbi.nlm.nih.gov/entrez/ query.fcgi?cmd $=$ Retrieve $\& \mathrm{db}=$ PubMed\&list uids $=$ $14551097 \&$ dopt $=$ Abstract

Tzeng WC, Lipson JG. The cultural context of suicide stigma in Taiwan. Qual Health Res 2004;14:345-58.

http://www.ncbi.nlm.nih.gov/entrez/ query.fcgi? $\mathrm{cmd}=$ Retrieve $\& \mathrm{db}=$ pubmed $\&$ dopt $=$ Abstract $\&$ list_uids $=15011902$

Wicke D, Coppin R, Payne S. Teamworking in nursing homes. J Adv Nurs 2004;45:197-204.

http://www.ncbi.nlm.nih.gov/entrez/

query.fcgi?cmd $=$ Retrieve $\&$ db $=$ PubMed\&list_uids $=$ 14706005\&dopt $=$ Abstract

Widerman E. Pathways to adult diagnosis of CF: the impact of pre-diagnosis experience on post-diagnosis responses and needs. Patient Educ Couns 2004:52:69-77.

http://www.ncbi.nlm.nih.gov/entrez/

query.fcgi?cmd $=$ Retrieve \&db $=$ PubMed\&list_uids $=$ $14729293 \&$ dopt $=$ Abstract

Wilkin K, Slevin E. The meaning of caring to nurses: an investigation into the nature of caring work in an intensive care unit. J Clin Nurs 2004;13:50-9.

http://www.ncbi.nlm.nih.gov/entrez/

query.fcgi?cmd $=$ Retrieve $\& \mathrm{db}=$ PubMed\&list_uids $=$ 14687293\&dopt $=$ Abstract

Yin LK, Twinn S. The effect of childhood cancer on Hong Kong Chinese families at different stages of the disease Cancer Nurs 2004;27:17-24. 